\title{
EGFR targeted therapy for lung cancer: are we almost there?
}

\author{
Giulio Metro \\ Medical Oncology, Santa Maria della Misericordia Hospital, Azienda Ospedaliera di Perugia, Perugia, Italy \\ Correspondence to: Giulio Metro, MD. Santa Maria della Misericordia Hospital, Azienda Ospedaliera di Perugia, via Dottori, 1, 06156 Perugia, Italy. \\ Email: giulio.metro@yahoo.com. \\ Provenance: This is an invited Editorial commissioned by Section Editor Hengrui Liang (Nanshan Clinical Medicine School, Guangzhou Medical \\ University, Guangzhou, China). \\ Comment on: Zhong WZ, Wang Q, Mao WM, et al. Gefitinib versus vinorelbine plus cisplatin as adjuvant treatment for stage II-IIIA (N1-N2) \\ EGFR-mutant NSCLC (ADJUVANT/CTONG1104): a randomised, open-label, phase 3 study. Lancet Oncol 2018;19:139-48.
}

Submitted Feb 23, 2018. Accepted for publication Mar 06, 2018.

doi: $10.21037 /$ tlcr.2018.03.11

View this article at: http://dx.doi.org/10.21037/tlcr.2018.03.11

In the era of precision medicine, predictive biomarkers have revolutionized the therapeutic approach to advanced non-small cell lung cancer (NSCLC) (1). So far, sensitizing epidermal growth factor $(E G F R)$ mutations (exon 19 deletion or exon $21 \mathrm{~L} 858 \mathrm{R})$ and anaplastic lymphoma kinase $(A L K)$ rearrangements are among the best described targetable alterations, against which highly effective small molecules tyrosine-kinase inhibitors (TKIs) have been developed. Unlike cytotoxic chemotherapy, TKIs are administered orally, and often provide a rapid and durable tumor response, which translate into better patient acceptance, greater efficacy, and improved quality of life compared to chemotherapy. Gefitinib, erlotinib, and afatinib were the first EGFR-TKIs to replace platinum-based chemotherapy as standard first-line treatment for patients with $E G F R$-mutant disease, while, more recently, the ALK-TKI crizotinib was shown to be far superior than platinum-pemetrexed as up-front therapy for $A L K$-positive advanced NSCLC (1). Nevertheless, virtually all patients treated with a TKI will eventually relapse, usually within one year. Importantly, as biological mechanisms that underlie resistance to treatment continue to emerge, second- and third-generation TKIs have been developed in order to effectively delay/prevent or overcome disease relapse. More in details, with regard to EGFR-mutant disease, resistance is due to the occurrence of a secondary mutation in exon 20 , namely T790M, in roughly $60 \%$ of cases. In this context, osimertinib has been recently shown to be more effective than platinum-pemetrexed as salvage treatment for EGFR T790M-positive disease (2). More recently, osimertinib was also shown to outperform an
EGFR-TKI (gefitinib or erlotinib) as first-line treatment for EGFR-mutant NSCLC (3).

Against this background, it is unclear whether an EGFR-TKI would maintain its remarkable efficacy as adjuvant treatment following complete tumor resection for early stage disease. At the present time, platinum-based chemotherapy should be considered as the standard therapy for patients with completely resected stage II-IIIA NSCLC, having convincingly demonstrated a survival improvement of approximately $5 \%$ at 5 years, regardless of whether an EGFR mutation was detected in tumor tissue $(4,5)$.

Recently, Zhong et al. reported the results of the phase 3 'ADJUVANT' trial, in which EGFR-mutant NSCLC patients with completely resected stage II-IIIA (N1$\mathrm{N} 2$ ) disease were randomly allocated to either standard platinum-based chemotherapy for four cycles (cisplatin $75 \mathrm{mg} / \mathrm{m}^{2}$ on day 1 plus vinorelbine $25 \mathrm{mg} / \mathrm{m}^{2}$ on days 1 and 8 , every 3 weeks) or gefitinib $250 \mathrm{mg}$ once daily for 2 years (6). Based on the assumption that cisplatin plus vinorelbine would provide a median disease free survival (DFS) of 31 months, it was calculated that 220 patients had to be randomized in order to detect a $40 \%$ improvement for DFS in favor of gefitinib [hazard ratio (HR) 0.6]. Importantly, health-related quality of life (HRQoL) was among key secondary end-points. Overall, 222 patients were randomized, 111 per group, of which 106/111 (95.5\%) and $87 / 111(78.4 \%)$ went on to receive at least a dose of study treatment in the gefitinib and chemotherapy arms, respectively. The results showed that the primary endpoint of DFS was met, as this was significantly longer for 
Table 1 Prospective adjuvant trials that compare directly an EGFR-TKI with platinum-based chemotherapy (no adjuvant chemotherapy allowed in the EGFR-TKI arm)

\begin{tabular}{|c|c|c|c|c|c|c|}
\hline Author, phase (ref.) & Population & $\begin{array}{l}\text { Stage, biomarker } \\
\text { selection }\end{array}$ & No. of pts, design & $\begin{array}{l}\text { Length of exposure } \\
\text { to EGFR-TKI (years) }\end{array}$ & $\begin{array}{l}\text { Primary } \\
\text { end-point }\end{array}$ & $\begin{array}{l}\text { Results for primary } \\
\text { end-point }\end{array}$ \\
\hline Zhong et al., 3 (6) & Asiatic & $\begin{array}{l}\text { II-IIIA(N1,N2)*, } \\
\text { EGFR mutation }{ }^{*}\end{array}$ & $\begin{array}{l}\text { 222, gefitinib vs. } \\
\text { cisplatin/vinorelbine }\end{array}$ & 2 & DFS & $\begin{array}{c}28.7 \text { vs. } 18.0 \text { months, } \\
H R=0.60 ; P=0.0054\end{array}$ \\
\hline Yue et al., 2 (7) & Asiatic & $\begin{array}{l}\text { IIIA* }{ }^{*} \text { EGFR } \\
\text { mutation }^{*}\end{array}$ & $\begin{array}{l}\text { 102, erlotinib vs. } \\
\text { cisplatin/vinorelbine }\end{array}$ & 2 & $\begin{array}{l}\text { 2-year } \\
\text { DFS rate }\end{array}$ & $\begin{array}{c}81.35 \% \text { vs. } 44.62 \% \\
\mathrm{P}<0.001\end{array}$ \\
\hline
\end{tabular}

${ }^{*}, 7^{\text {th }}$ TNM staging; ${ }^{*}$, exon 19 deletion or exon 21 L858R. DFS, disease-free survival; EGFR-TKI, epidermal growth factor receptor-tyrosine kinase inhibitor; HR, hazard ratio; No., number; OS, overall survival; pts, patients; vs., versus; ref., reference.

patients assigned to gefitinib than for those assigned to platinum-based chemotherapy (median 28.7 months, 95\% CI: 24.9-32.5, versus 18.0 months, 13.6-22.3; HR 0.60, 95\% CI: $0.42-0.87 ; \mathrm{P}=0.0054)$. While overall survival was not yet mature at the time of the analysis, both safety and HRQoL significantly favored gefitinib. In fact, any grade adverse events occured in $58 \%$ of patients in the gefitinib arm versus $80 \%$ of patients in the chemotherapy arm. Also, the most commonly reported grade $\geq 3$ adverse events in gefitinib-treated patients were hypertransaminasemia ( $2 \%$ versus none for chemotherapy), while in patients who received chemotherapy they were neutropenia (34\% versus none), leucopenia (16\% versus none for gefitinib), and vomiting ( $9 \%$ versus none). In addition, HRQoL provided significantly higher scores for patients who received gefitinib compared to those who received cisplatin plus vinorelbine. On this basis, the authors concluded that "gefitinib could be considered a treatment option for EGFRmutant NSCLC patients".

To put these results into context, it is worth mentioning the results of a recently reported randomized phase 2 study named 'EVAN', which, similarly to the 'ADJUVANT' trial, was conducted in an Asiatic population at high risk of relapse (stage IIIA). The 'EVAN' trial allocated EGFRmutant patients to either cisplatin plus vinorelbine for four cycles (same schedule as the 'ADJUVANT' trial) or an EGFR-TKI, namely erlotinib, for 2 years (7). Similarly to the 'ADJUVANT' trial, the results of the 'EVAN' study demonstrated a longer DFS in favor of an EGFR-TKI compared to chemotherapy (median 42.4 months, $95 \%$ CI: 31.6-NR, versus 20.9 months, 12.2-32.3; HR 0.26, 95\% CI: $0.13-053 ; \mathrm{P}<0.001)$ as well as an improved safety profile for erlotinib-treated patients. Remarkably, although overall survival data were still immature, a trend toward a survival improvement was shown in the erlotinib arm. Therefore, based on the consistent results across the 'ADJUVANT' and
'EVAN' trials, it can be reasonably concluded that EGFRmutant Asiatic patients with completely resected NSCLC who are at high risk of relapse may benefit from an adjuvant EGFR-TKI (Table 1).

Nevertheless, while confirmatory studies are underway in non-Asiatic and lower risk patients, it should be questioned whether overall survival as opposed to DFS should be regarded as the most important outcome of benefit in clinical trials aimed at assessing the effectiveness of adjuvant therapy for completely resected NSCLC patients. In 2013, a re-analysis of two meta-analyses of individual patients' data from trials of adjuvant chemotherapy suggested that there was a strong correlation between DFS and overall survival in early stage NSCLC patients, thus supporting the use of DFS as a surrogate end-point for overall survival (8). However, it is uncertain whether this correlation will hold true also in EGFR-mutant patients treated with an adjuvant EGFR-TKI. In fact, an EGFR-TKI may not be able to show a significant improvement in survival due to the fact that many of the patients who relapse in the standard chemotherapy arm will still experience prolonged survival from highly effective post-recurrence therapies, which will likely dilute the impact on survival of an adjuvant EGFR-TKI in the experimental arm. With regard to this, a retrospective study performed in over one-hundred $E G F R$-mutant advanced NSCLC patients treated with either gefitinib or erlotinib in any line found a considerable median survival and 5-year survival of 30.9 months and $14.6 \%$, respectively (9). Thus, it could be fair to say that some EGFR-mutant early stage disease patients may still benefit from an adjuvant EGFR-TKI because of increased DFS, improved safety, and better quality of life, even in the absence of a survival advantage.

Similarly, another reason why an adjuvant EGFRTKI may not improve overall survival is that such a therapy is typically associated with a cytostatic rather than 
cytotoxic effect, which does not allow full eradication of micrometastatic disease. Accordingly, in the 'ADJUVANT' trial Kaplan-Meier curve for DFS underwent clear separation at around 12 months and then came together at about 36 months (approximately 12 months after stopping gefitinib), with no apparent tail of non-progressors in either treatment arm. Indeed, similar rates of disease relapse were observed in this trial for both gefitinib and chemotherapy in the intention-to-treat population ( $52 \%$ versus $50 \%$, respectively). Of note, similarly shaped curves for DFS were observed in the subgroup of EGFR-mutant NSCLCs treated within the 'RADIANT' trial, in which patients were allocated to either erlotinib or placebo for 24 months following completion of adjuvant platinum-based chemotherapy (10). These data suggest that, compared to chemotherapy, an EGFR-TKI might just delay but not prevent disease relapse, which occurs in most cases anyway upon discontinuation of the drug after 24 months of treatment. On this basis, it can be hypothesized that some patients may benefit from continuation of an EGFR-TKI far beyond 24 months, although detractors will argue that the inherent benefits of a protracted EGFR-TKI treatment should be weighed against its potential adverse events. However, because symptomatic recurrence has a significant impact on both patient morbidity and costs to society, alive on therapy is not equivalent to alive on therapy free of disease. With this in mind, identifying the patients who may benefit from indefinite continuation of an EGFR-TKI beyond 24 months because of a higher risk of relapse would be crucial in order to maximize control of microscopic disease in selected cases. In a study of stage I-III lung cancer patients treated with curative intent, Chaudhuri et al. found that circulating tumor DNA could be a reliable surrogate marker for the identification of patients who will relapse after treatment (11). Whether other biomarkers and/or techniques will be developed in order to personalize adjuvant treatment at early time points is the matter of ongoing research.

Clinical research on whether patients with completely resected EGFR-mutant NSCLC should receive an EGFRTKI in the adjuvant setting is just at the beginning. In the future, when the results of ongoing studies will be available, a meta-analysis of all available data could be performed in order to unveil a survival benefit in favor of an adjuvant EGFR-TKI. However, in the era of third-generation EGFR-TKIs for advanced disease, it would also be important to test such agents in the adjuvant setting as well. At the present time, the 'ADAURA' study (NCT02511106) is currently randomizing patients with $E G F R$-mutant stage IB-IIIA NSCLC to either osimertinib or placebo for 2 years after platinum-based chemotherapy, with DFS being the primary end-point. Hopefully, this and other trials with novel molecules will live up to their expectation and ultimately demonstrate a relevant survival benefit.

\section{Acknowledgements}

None.

\section{Footnote}

Conflicts of Interest: The author has no conflicts of interest to declare.

\section{References}

1. Toschi L, Rossi S, Finocchiaro G, et al. Non-small cell lung cancer treatment (r)evolution: ten years of advances and more to come. Ecancermedicalscience 2017;11:787.

2. Mok TS, Wu YL, Ahn MJ, et al. Osimertinib or PlatinumPemetrexed in EGFR T790M-Positive Lung Cancer. N Engl J Med 2017;376:629-40.

3. Soria JC, Ohe Y, Vansteenkiste J, et al. Osimertinib in Untreated EGFR-Mutated Advanced Non-Small-Cell Lung Cancer. N Engl J Med 2018;378:113-25.

4. Pignon JP, Tribodet H, Scagliotti GV, et al. Lung adjuvant cisplatin evaluation: a pooled analysis by the LACE Collaborative Group. J Clin Oncol 2008;26:3552-9.

5. Burdett S, Pignon JP, Tierney J, et al. Adjuvant chemotherapy for resected early-stage non-small cell lung cancer. Cochrane Database Syst Rev 2015;(3):CD011430.

6. Zhong WZ, Wang Q, Mao WM, et al. Gefitinib versus vinorelbine plus cisplatin as adjuvant treatment for stage II-IIIA (N1-N2) EGFR-mutant NSCLC (ADJUVANT/ CTONG1104): a randomised, open-label, phase 3 study. Lancet Oncol 2018;19:139-48.

7. Yue D, Xu S, Wang Q, et al. Efficacy and safety of erlotinib vs vinorelbine/cisplatin as adjuvant therapy for stage IIIA EGFR mutant NSCLC patients. J Thorac Oncol 2017;12:abstr S1789.

8. Mauguen A, Pignon JP, Burdett S, et al. Surrogate endpoints for overall survival in chemotherapy and radiotherapy trials in operable and locally advanced lung cancer: a re-analysis of meta-analyses of individual patients' data. Lancet Oncol 2013;14:619-26.

9. Lin JJ, Cardarella S, Lydon CA, et al. Five-Year Survival in 
EGFR-Mutant Metastatic Lung Adenocarcinoma Treated with EGFR-TKIs. J Thorac Oncol 2016;11:556-65.

10. Kelly K, Altorki NK, Eberhardt WE, et al. Adjuvant Erlotinib Versus Placebo in Patients With Stage IBIIIA Non-Small-Cell Lung Cancer (RADIANT): A Randomized, Double-Blind, Phase III Trial. J Clin Oncol

Cite this article as: Metro G. EGFR targeted therapy for lung cancer: are we almost there? Transl Lung Cancer Res 2018;7(Suppl 2):S142-S145. doi: 10.21037/tlcr.2018.03.11
2015;33:4007-14.

11. Chaudhuri AA, Chabon JJ, Lovejoy AF, et al. Early Detection of Molecular Residual Disease in Localized Lung Cancer by Circulating Tumor DNA Profiling. Cancer Discov 2017;7:1394-403. 